\title{
Unterschiede in der Blütezeit einiger Frühlingspflanzen der Umgebungen Ragusa's.
}

\author{
Von Prof. E. Nikolić (Ragusa).
}

Obwohl die strenge Kälte, die sich heuer überall fühlbar machte, in Ragusa kaum zu spüren war, zeigte sich doch in der Entwicklung und Blütezeit der Frühlingspflanzen eine auffallende Verspätung, die am deutlichsten ersichtlich wird, wenn man die heurigen phänologischen Beobachtungen mit jenen günstigerer Jahre vergleicht.

Ich habe hier nur die Daten zweier Jahre vorgeführt (1888 und 1895); die zwischen diesen Grenzen liegenden Daten könmen vielleicht für speciellere klimatologische Studien von Belang sein; hier aber als überflüssig, wurden sie einfach nicht berücksichtigt.

\begin{tabular}{|c|c|c|c|}
\hline \multirow{2}{*}{ Pfla $\mathrm{nze}$} & \multirow{2}{*}{ Ort } & \multicolumn{2}{|c|}{ Tag der ersten Blüte } \\
\hline & & 1888 & 1895 \\
\hline Cercis siliquastrum L.......... & Gravosa & $2 \%$ & $16 / 4$ \\
\hline Cytisus infestus Guss........... & Mad. delle Grazie & $10 \%$ & $28 / 9$ \\
\hline Ononis antiquorum L. ... & Annunciata & $10 / 5$ & $\begin{array}{c}\text { derzeit }(15 / 5) \\
\text { nooh nicht } \\
\text { bluhend }\end{array}$ \\
\hline Anthyllis Barba Jovis L. ... & $n$ & $18 / 4$ & $2 / 5$ \\
\hline Coronilla stipularis Lam. ... & San Giacomo & $13 / 8$ & $28 / 3$ \\
\hline Acacia Jutinbrissin Willd.... & Bella vista & $8 / 6$ & $\begin{array}{l}\text { noch keine Spur } \\
\text { von Belaubung }\end{array}$ \\
\hline Amygdalus communis L. ... & San Giacomo & $28 / 19 \quad 1877$ & $26 / 1$ \\
\hline Matthiola incana R. Br. & Dauce & $12 / 1$ & $26 / 2$ \\
\hline$" \quad$ tristis R. Br. ...... & Goriza San Biagio & $2 / 2$ & $28 / 3$ \\
\hline Cheiranthus Cheini L..... & Bella vista & $1 \%$ & $12 \%$ \\
\hline Phlomis fruticosa L. .... & San Giacomo & $28 / 4$ & $2 / 5$ \\
\hline Prasium majus L. ........... & n & $1 / 4$ & $15 / 4$ \\
\hline Convolvulus lucorum L...... & Lapad & $15 / 5$ & $\begin{array}{l}\text { noch nicht } \\
\text { bluhend }\end{array}$ \\
\hline$" \quad$ althaeoides ...... & San Giacomo & $30 / \mathrm{s}$ & $10 \%$ \\
\hline Viburnum Tinus L, ......... & um die Stadt & $14 / 1$ & $25 / 2$ \\
\hline $\begin{array}{l}\text { Erodium cicutarium var. } \\
\text { acaulis (?) ................... }\end{array}$ & Lapad & $1 \%$ & $12 / 2$ \\
\hline Putoria Calabrica Pers....... & San Giacomo & $25 / 4$ & $2 / 5$ \\
\hline Tilia europaea Willd........... & Pille & $6 / 5$ & $15 / 5$ \\
\hline Plantago psyllium ............ & San Giacomo & $26 / 4$ & $28 / 4$ \\
\hline
\end{tabular}




\begin{tabular}{|c|c|c|c|}
\hline \multirow{2}{*}{$P$ flanze } & \multirow{2}{*}{ Ort } & \multicolumn{2}{|c|}{ Tag der ersten Blüte } \\
\hline & & 1888 & 1895 \\
\hline Punica granatum............... & Gravosa & $18 / 5$ & noch nicht \\
\hline Osyris alba $\mathrm{L}$. .................... & San Giacomo & $2 \%$ & $1 / 5$ \\
\hline Cotyledon Umbilicum L....... & $"$ & $1 / 5$ & $1 / 5$ \\
\hline Euphorbia dendroides L. ... & " & $11 / 2$ & $24 / 2$ \\
\hline Melia Azedarach L.............. & Pille & $8 / 5$ & $\begin{array}{c}(15 / 5) \text { laum die } \\
\text { Blutenknospen } \\
\text { angedertet }\end{array}$ \\
\hline Solanum Sodomeum L. ....... & Mad. delle Grazie & $30 / 4$ & $15 / 5$ \\
\hline Frawinus Ornus L. ............. & Ombla & $15 / 4$ & $3 i / 4$ \\
\hline Anemone stellata L.............. & Gravosa & $20 / 5$ & $28 / 9$ \\
\hline Smilax mauritaniea Poir. ... & Breno & $20 / 5$ & $\begin{array}{l}\text { noch nicht } \\
\text { blühend. }\end{array}$ \\
\hline Tamarix galliea L............. & Gravosa & $4 / 4$ & $2 \%$ \\
\hline , Africana Poir. ... & Pille & $10 / 4$ & $13 / 4$ \\
\hline Pinus Halepensis Miller...... & Petka & $30 / 3$ & $12 / 4$ \\
\hline Juniperus oxycedrus L. L..... & Montovierna & $2 / 8$ & $12 / 3$ \\
\hline Cupressus sempervirens $L \ldots$ & Pille & $2 / 2$ & $8 / 2$ \\
\hline$" \quad$ "divaricata & San Giacomo & $2 / 2$ & $\eta / 2$ \\
\hline Phoenix dactylifera L. ...... & " & $26 / 2$ & $12 / 3$ \\
\hline Opuntia Ficus Indica Mill. . & Montovierna & $30 / 4$ & $\begin{array}{l}\text { noel niwht } \\
\text { blithend }\end{array}$ \\
\hline n Amyclaea Ten....... & Aequedatto & $2 / 5$ & $"$ \\
\hline
\end{tabular}

Diese phänologischen Beobachtungen stimmen mit denen der "Flora Jadrensis" yon A. Alschinger, mit denen des "Botanischen Wegweisers" von Prof. Franz Petter und mit denen der "Plantae lignosae imperii Austriaci“ von Dr. Alois Pokorny nicht immer überein. So z. B. gibt Pok orny für die zwei Matthiola-Arten Juni und Juli als Blütezeit an und für Cheiranthus Mai und Juni. Pokorny und ebenso Alschinger geben für Olea europec ebenfalls Juni und Juli als Zeit der ersten Blüte an, wäbrend in Ragusa die Olea spätestens Mitte Mai schon in voller Blüte steht. Nach Pokorny soll Cistus solviaefolius L. im Mai und Juni zui Blüte kommen; hener war er Mitte April hier (Lapad) schon blühend.

Tamarix gallica und $T$. Africana blühen nach Pokorny im Juni bis August, hier in Ragusa aber Mitte und Ende April. Auch Punica granatum und Melia Azedarach sollen nach obigen Angaben im Juni und August blühen, während beide Ende Mai schon in voller Blüte stehen.

Ficus carica L. soll nach Pokorny und Bertoloni im August und September blühen; die cultivirte Species blüht aber im 
Juni und October. Die ersten Blütenknospen auf den androgynischen Individuen (Caprificus leucocarpa Gasparr.), zeigen sich hier schon mit den ersten Jännertagen und Ende März sind sie vollkommen entwickelt.

Bei dieser Gelegenheit möchte ich erwähnen, dass in I)almatien die Caprification weder geübt noch bekannt ist, wäbrend die alten Griechen und die hentigen Bewohner des Archipelagus und Calabriens diese Operation für einige Ficus-Sorten unbedingt für nothwendig halten.

Ragusa, am 15. Mai 1895.

Arbeiten des botanischen Institutes der k. k. deutschen Universität Prag. VII.

\section{Beitrag \\ zur Kenntnis der Gattung Alectorolophus All.}

Von Dr. Jacob von Sterneck (Prag).

(Mit 4 Tafeln [IV, VI, VII, XI] und 1 Karte.)

(Fortsetzung. ")

Werfen wir nun noch einen Blick auf die Veränderungen, die das Aufhören der Glacialzeit bei den ,alpini“ bewirkte. Diese waren vordem von den thalbewohnenden Arten (Typus des Freynii-goniotrichus) hauptsächlich durch die Corollenform verschieden; sie mochten demnach dem heutigen $A$. pulcher recht ähnlich gesehen baben. Bei Rücktritt der Eiszeit wurde diese Art auf die höchsten Erhebungen zurückgedrängt und so geschah es, dass sich ihr ehedem zusammenhängendes Areale theilte, und in jedem Stücke sich die Art den nenen Lehensbedingungen entsprechend anpasste. Auf diese Weise dürfte sich der Typus des $A$. lanceolatus vom wohl viel älteren - d. h. unverändert gebliebenen -- A. pulcher differenzirt haben.

Möglicherweise ist eine ähnliche Bildung dem A. dinaricus zu Grunde zu legen, jedoch sprechen andererseits die eigenthümliche Corollengestalt wieder für ein höheres Alter. Ich habe daher diese Zusammengehörigkeit nur als fraglich (smson) in das Schema (s. Nr. 10, S. 378) aufgenommen.

In der Zeit nach Ablauf der letzten diluvialen Eiszeit war somit die Bildung sämmtlicher bisher besprochenen Typen vollzogen, deren Verbreitungsgebiete sich mehr oder weniger geographisch ausschliessen, je nachdem die Ursachen ihrer Entstehung noch fortbestehen, beziehungsweise weggefallen sind.

Ich will daher zunächst deren gegenwärtiges Verbreitungsgebiet mittelst der nachstehenden Karte veranschaulichen, bemerke

1) Vergl. Nr. 10, S. 377. 